\title{
Axial phase measurements of light interacting with microstructures
}

\author{
Myun-Sik Kim, Toralf Scharf, and Hans Peter Herzig \\ Optics \& Photonics Technology Laboratory, Ecole Polytechnique Fédérale de Lausanne (EPFL), \\ Neuchâtel, CH-2000, Switzerland
}

\begin{abstract}
We present an experimental method to study field structures of highly confined light after interaction with microstructures. A high-resolution interference microscope (HRIM) allows us to measure the three-dimensional (3D) amplitude and phase distributions of light emerging from the sample. While the amplitude fields represent conventional pictures of light confinements like a hotspot, the phase fields exhibit peculiar behaviors, which are of significant interest. Longitudinal-differential interferometry can directly visualize and quantify phase deviations in 3D space with respect to a plane wave of the same frequency serving as a reference. The phase fields near the confinement exhibits particular phase features, e.g., axial phase anomaly and superluminal phase velocity. As example of the light interaction with microstructures, two specific optical phenomena have been investigated here: Gouy phase anomaly in the photonic nanojet and superluminal phase propagation of the spot of Arago. For the first time, we could experimentally demonstrate high-resolution axial phase measurements of such phenomena generated by microstructures of wavelengthscale size and at visible light with 642-nm wavelength.
\end{abstract}

Keywords: Gouy phase anomaly, axial phase shift, superluminal phase velocity, photonic nanojet, the spot of Arago, high-resolution interference microscope, longitudinal-differential interferometry

\section{INTRODUCTION}

Anomalous and peculiar features of axial phase fields in the vicinity of light confinement have been drawing our attention. In 1890 Gouy discovered that when a converging diffracted spherical wave passes through a focus, the axial phase exhibits an anomalous $\pi$ shift, which is named Gouy phase shift or anomaly in honor of his discovery ${ }^{1}$. The origin of this phenomenon has been studied, for example, with scalar and vector diffraction theories ${ }^{2-5}$ and with the concept of a Berry's geometric phase ${ }^{6}$. Such anomalous phase evolutions are observed not only in a focusing of a Gaussian beam but also in all other kinds of light confinements, for example, in photonic nanojets ${ }^{7}$ and in the spot of Arago ${ }^{8}$. Most interferometric studies imitate Gouy's original experiment ${ }^{1}$. The three dimensional optical field structures in close vicinity to the confinement region, for the best of our knowledge, were first reported in Ref. 7. Often a small-wavelength approximation assuming $\lambda<<r$ and high Fresnel number (FN) measurement system with $\lambda<<r$ and/or $r<<z$ are applied to experimental studies, where $\lambda$ is the operating wavelength, $r$ is the radius of the aperture or object, and $z$ is the distance between the observation plane and the object. In earlier measurements of photonic nanojets $[r=2 \mu \mathrm{m} \text { and } \lambda=642 \mathrm{~nm}]^{7}$, the observation plane was close to the object and the experimental conditions fall out of the aforementioned classical approximations. Eventually, such a case leads to low FN. Note that although the diameter $d(=2 r)$ of the sphere was of wavelength-scale $[d=3 \lambda]$, the existence of the Gouy phase anomaly could be demonstrated.

In our study we introduce a new measurement technique called longitudinal-differential interferometry ${ }^{9}$ in order to investigate axial phase behaviors when light interacts with microstructures. We are especially interested in situations when wavelength-scale objects are studied at low FN conditions, a case usually avoided for simplicity of theoretical analysis. We will present the experimental setup and the working principle of longitudinal-differential interferometry. As examples of the light interactions with microstructures, two particular optical phenomena are considered. One is a scattering effect of a dielectric microsphere under plane-wave illumination, which leads to a strong hotspot on the shadow-side surface of the sphere called the photonic nanojet ${ }^{10}$. The other is a diffraction effect of a circular opaque obstacle (e.g. a metallic disc), which generates a hotspot in the shadow of the obstacle named the spot of Arago ${ }^{11,12}$. The axial phase shifts eventually lead to the on-axis local superluminal phase propagation ${ }^{8,13}$. This kind of superluminal wave propagation in air can also be observed in Bessel beams ${ }^{14}$. We experimentally demonstrate the existence of the Gouy phase anomaly in the photonic nanojet and the superluminal phase propagation of the spot of Arago.

*myunsik.kim@epfl.ch; phone 41-32-718-3279; fax 41-32-718-3201; http://opt.epfl.ch/

Reliability, Packaging, Testing, and Characterization of MEMS/MOEMS and Nanodevices XI,

edited by Sonia M. García-Blanco, Rajeshuni Ramesham, Proc. of SPIE Vol. 8250, 825007

(C) 2012 SPIE · CCC code: 0277-786X/12/\$18 - doi: 10.1117/12.905461

Proc. of SPIE Vol. $8250825007-1$ 


\section{EXPERIMENTAL SETUP}

Longitudinal-differential interferometry is implemented in our basic setup, the high-resolution interference microscope. The HRIM is a kind of holographic microscopy in a sense of recording interferograms and retrieving information of light fields of interest, especially amplitude and phase distributions. As it relies on interferometry, such as a Mach-Zehnder interferometer and phase shifting interferometry, we have named our experimental system an interference microscope. In general, interferometric testing systems have been developed for two-dimensional (2D) measurements, for example, surface profiling and wavefront measurements of light fields in a plane of interest ${ }^{15}$. The major difference from other types of interference microscopes is the capability to record real 3D amplitude and phase fields by scanning the samples along the axial direction.

The HRIM has already been proven as a powerful tool for the 3D characterizations of macro-, micro-, and nano-optical elements $^{16-20}$ and to measure light field features with nanometric precision ${ }^{21}$. The HRIM operates in transmission with inline geometry by employing a Mach-Zehnder interferometer as shown in Fig. 1(a). A single mode polarized laser diode (CrystaLaser, $642 \mathrm{~nm}$ : DL640-050-3) is employed as light source, which is expanded and collimated by a spatial filtering technique. This illumination plane wave, which is normally incident upon the sample, propagates along the positive $z$ axis and is polarized in the $x$-direction. In the classical interferometric arrangement a polarizing beam splitter (BS1) divides intensities in a reference and an object arm with adjustable energy ratio. Half wave plates (HWP) and GlanTaylor (G-T) polarizers are used to adjust the intensities and to optimize the contrast of the interference fringes. In the reference arm, a piezo-electrically driven mirror is mounted to change the optical path length. The phase distribution in a single plane of the wave field is obtained by measuring the interference fringes at different mirror positions and employing the well-known 5-frame algorithm, which is called Schwider-Hariharan method ${ }^{22,23}$. Five frames of the intensity pattern, from which the 2D phase information can be directly retrieved, are recorded with each frame being shifted by adding an optical path of $\lambda / 4$ or $0.5 \pi$. The sample is mounted on a precision piezo stage with a $z$-scan range of $500 \mu \mathrm{m}$ and a nominal accuracy of $1 \mathrm{~nm}$ (MAD LAB CITY, NANO Z500). This $z$-axis piezo stage is used to precisely define the plane of interest at the highest resolution and measure 3D light fields emerging from the wavelength-scale objects by scanning the sample along the axial direction.

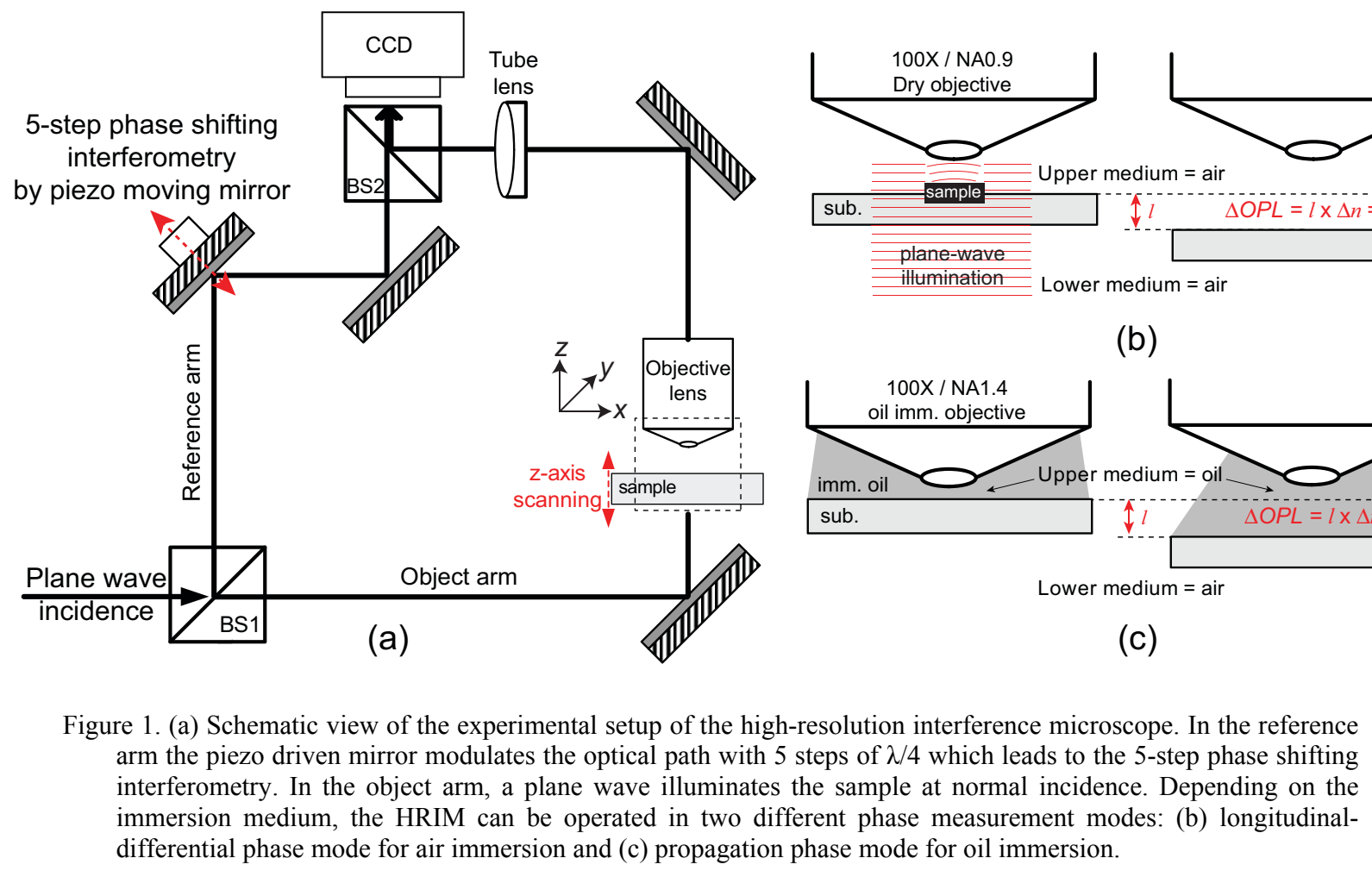


As shown in Fig. 1, the scanning of the sample along the optical axis, which is aimed to record the 3D field structures, leads to two particular observation conditions depending on the immersion medium: Fig. 1(b) for air and Fig. 1(c) for oil. Imagine the object space between the substrate and the observing objective (a 100X / NA 0.9 dry objective or a 100X / NA1.4 oil immersion objective -Leica Microsystems) has a refractive index $n_{\text {upper }}$ and below the substrate $n_{\text {lower }}$ as depicted in Figs. 1(b) and (c). The variation of the optical path length (OPL) in the object arm is then given as

$$
\triangle O P L=\left(n_{\text {upper }}-n_{\text {lower }}\right) \cdot l,
$$

where $l$ is the scanning length along the $z$-axis. In Fig. 1(b), when the upper and lower medium are the same, $\Delta \mathrm{OPL}$ equals zero due to the vanishing of the refractive index difference $\Delta \mathrm{n}\left(=\mathrm{n}_{\text {upper }}-\mathrm{n}_{\text {lower }}\right)=0$. This means that during the $z$ axis scanning the phase of the plane wave remains constant and that of the perturbed wave is measured as the deviation from that constant phase, which is the self-reference; in this way a longitudinal-differential phase is measured.

Considering now the case where the upper space is filled with a different medium. In the oil immersion scheme of Fig. 1 (c), the refractive index difference $\Delta \mathrm{n}\left(=\mathrm{n}_{\text {upper }}-\mathrm{n}_{\text {lower }}\right)$ is non-zero. Therefore, Eq. (1) varies and this leads to an optical path difference (OPD) between reference and object arms, which causes the phase wrapping along the axial direction. The period of this phase wrapping is inversely proportional to $\Delta \mathrm{n}$, which is approximately two times larger than the operation wavelength in air with $\mathrm{n}_{\mathrm{oil}}=1.5$ and $\mathrm{n}_{\text {air }}=1$. The phase distributions measured in this manner imitates the phase of a propagating light with an elongated wavelength. Note that this scheme is explained only for reference and to highlight the longitudinal-differential phase features of the air immersion. For more details of longitudinal-differential interferometry, a graphical representation is given in Fig. 1(b) that shows two waves in the space between the substrate and the objective: one is a part of the unperturbed illumination plane wave propagating out of the sample and the other is the perturbed wave by the sample. The longitudinal-differential phase distribution of the unperturbed plane wave appears as constant while that of the perturbed wave is imaged as a difference. With the concept of longitudinal-differential interferometry one can measure the axial phase evolution as the difference between an unperturbed plane wave (selfreference wave) and a perturbed wave (object wave) directly.

\section{GOUY PHASE IN PHOTONIC NANOJET}

As we discussed in the introduction, a single dielectric microsphere generates a highly localized confinement of light under a plane-wave illumination, which is called the photonic nanojet ${ }^{10}$. This kind of light confinement originates from the Mie scattering of a dielectric microsphere, whose size is larger than the wavelength ${ }^{24,25}$. The longitudinal-differentialmode HRIM records the 3D amplitude and phase distributions near the photonic nanojet spot, in our case created by a 2 $\mu \mathrm{m}$ glass sphere at $642-\mathrm{nm}$ illumination. The propagating plane wave far away from the sphere serves as the in situ selfreference wave and the differential phase shift is directly measured. During the measurements, phase drifts along the $z$ axis are inevitable but could be directly corrected by assuming a constant phase of the self-reference plane wave. Figures 2(a) and 2(b) show measured longitudinal intensity and longitudinal-differential phase maps for the 2- $\mu \mathrm{m}$ sphere (white circle).

Even though the origin and mechanism of the confinement is different, the intensity distribution of the nanojet mimics that of an aplanatic focusing, i.e. a point focus. The longitudinal-differential phase distribution visualizes and quantifies how much the phase of the nanojet spot differs from that of the unperturbed plane wave, which is shown as a constant (uniform color) outside the sphere. For the detailed investigation of the axial phase behavior, we extract the on-axis longitudinal-differential phase data from four different measurements with the same object. These data are shown in Fig. 2(c). The $z=2 \mu \mathrm{m}$ plane corresponds to the center of the nanojet spot, where the peak intensity occurs.

Three different regimes can be distinguished along the $z$-axis.

(1) Positions close to the substrate $[z=0-1 \mu \mathrm{m}]$ : The observation is influenced by aberrations and there are light fields suffering from multiple reflections between the substrate and the sphere, which cause experimental uncertainties.

(2) Positions inside the sphere, at the intensity hotspot and behind the intensity hotspot $[z=1-3.5 \mu \mathrm{m}]$ : The high contrast of interference fringes and small amount of aberrations provide reliable results. Theory and measurement show excellent agreement in this region, as expected [7].

(3) Positions far away behind the hotspot $[z=3.5-5 \mu \mathrm{m}]$ : As seen in Fig. 2(a), there is almost no intensity detectable and measured phases are subjected to noise. 

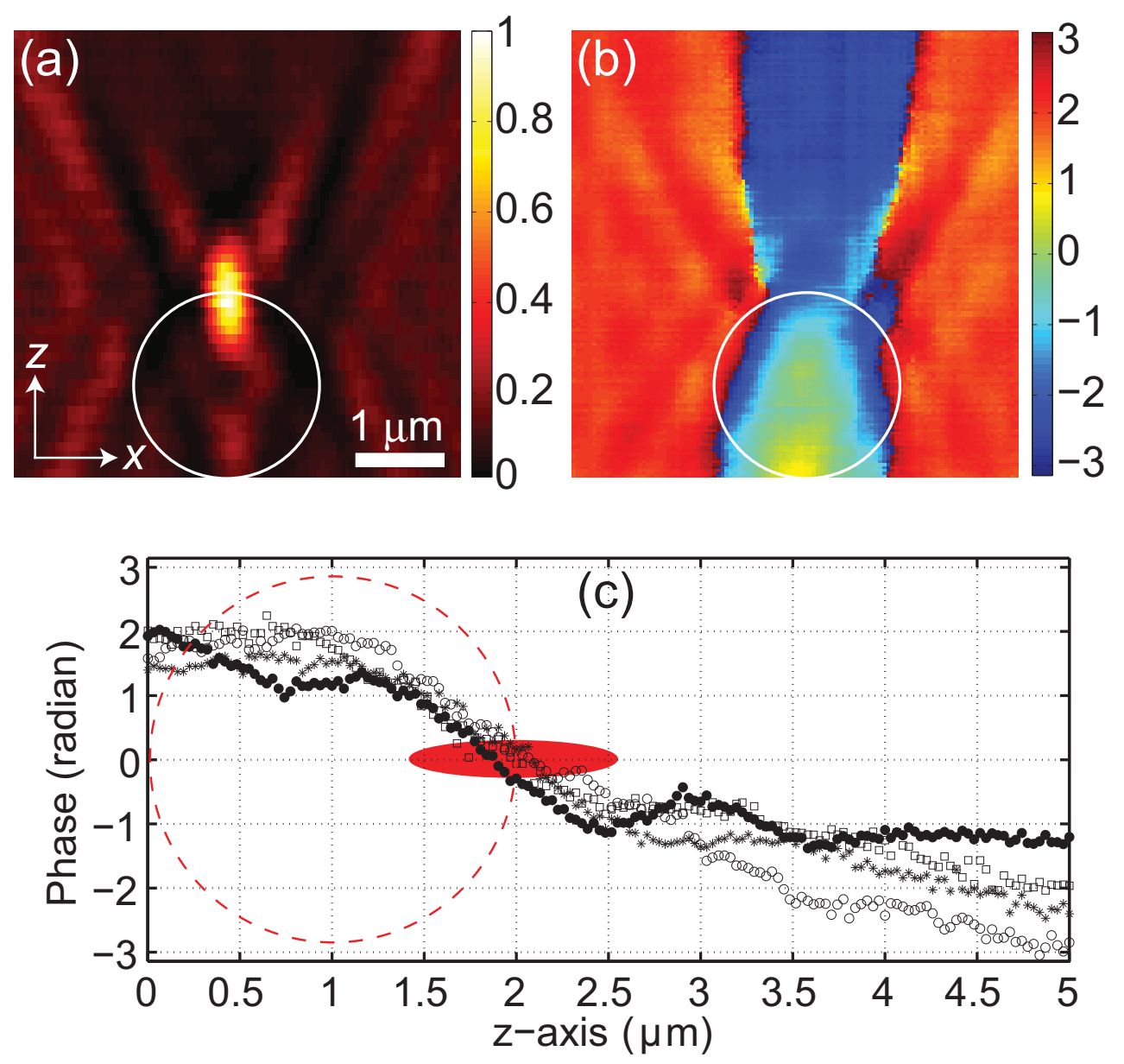

Figure 2. (Color online) The measured longitudinal $(x-z)$ light field distributions of the photonic nanojet created by a 2$\mu \mathrm{m}$ glass sphere: (a) the intensity, (b) the longitudinal-differential phase. The intensity is normalized and the white circle indicates the $2-\mu \mathrm{m}$ sphere. (c) The on-axis longitudinal-differential phase data from four different measurements, where the center of the nanojet locates in the plane $z=2 \mu \mathrm{m}$. In the vicinity of the nanojet spot $[z=$ $1.5-2.5 \mu \mathrm{m}]$, we can observe approximately $\pi$ phase shift, which corresponds to the Gouy phase anomaly of a point focusing.

Nevertheless, the excellent agreement between simulation and experiment, found in Ref. 7, provides us confidence that the results are sufficiently exact. In Fig. 2(c), the longitudinal-differential phase data shows approximately a $\pi$ phase shift in the vicinity of the nanojet focus (the second regime). This proves that the field behavior of the photonic nanojet is analogous to that of the highly focused beam and demonstrates the existence of the Gouy phase in the photonic nanojet.

\section{SUPERLUMINAL PHASE VELOCITY OF ARAGO SPOT}

When a circular obstacle like a metallic disc is illuminated, a bright spot appears in its shadow, which is named the spot of Arago or Poisson's bright spot ${ }^{11,12}$. It is the result of the constructive interference of waves diffracted at a circular symmetric edge of the obstacle. Since the cylindrical symmetry is a key of such an effect, we restrict our attention to onaxis observation points to analyze the phase shift and the superluminal behavior. Figure 3 shows the measured longitudinal intensity and phase distributions recorded by the longitudinal-differential-mode HRIM. The case when a plane wave shines at normal incidence on a 10- $\mu \mathrm{m}$ metallic disc from below (along the positive $z$-direction) is shown. While the intensity distribution in Fig. 3(a) does not show much information except the existence of the bright spot in the shadow of the obstacle, the longitudinal-differential phase distribution in Fig. 3(b) reveals more detailed features in the spatial domain, where only low intensities are detectable, and a particular behavior along the optical axis. 

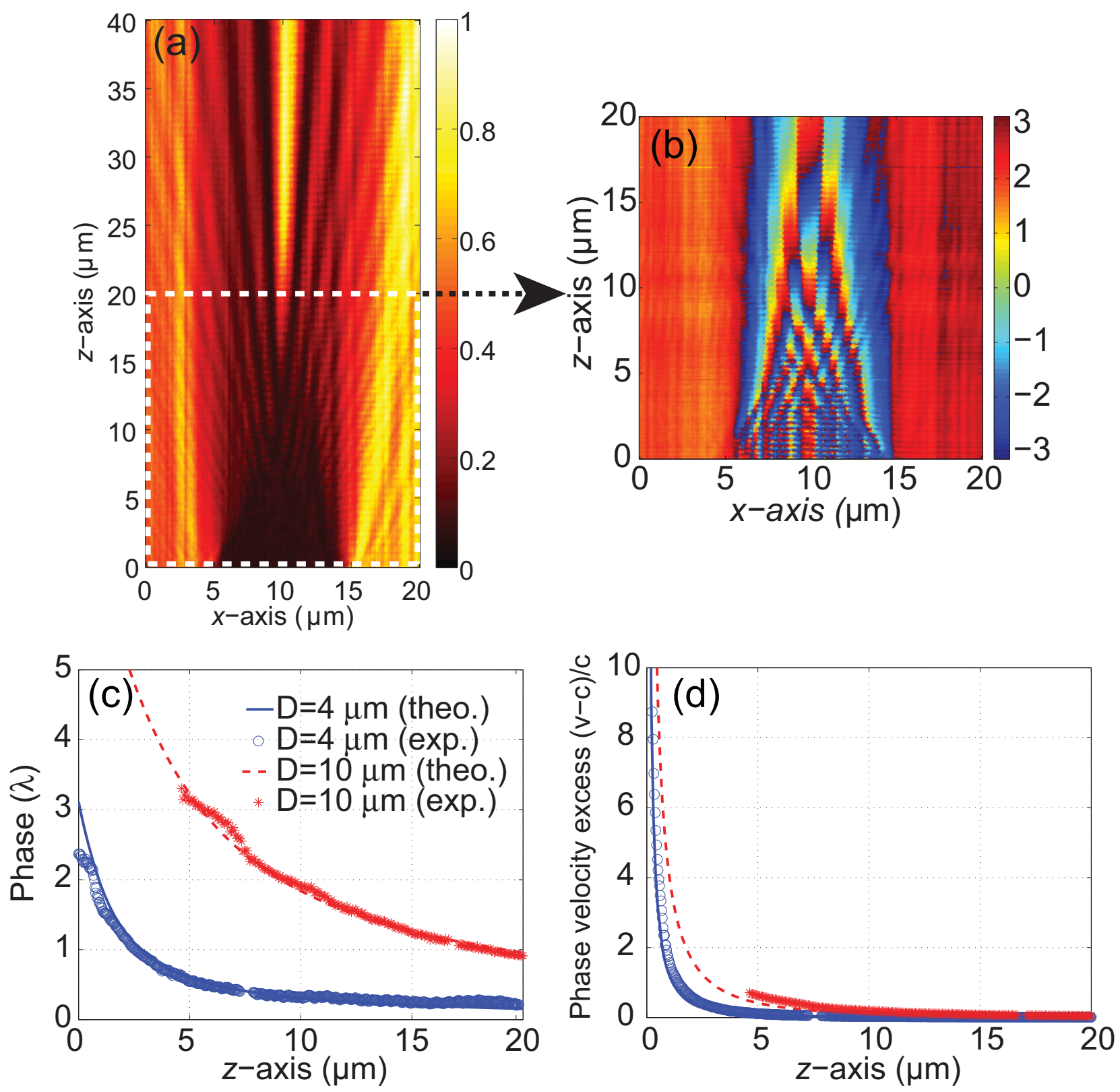

Figure 3. (Color online) The measured longitudinal $(x-z)$ light field distributions of the spot of Arago generated by a 10$\mu \mathrm{m}$ metallic disc: (a) the intensity, (b) the longitudinal-differential phase. The phase is measured close to the disc as indicated in the white dashed square in (a) $[z=0-20 \mu \mathrm{m}]$. (c) The on-axis longitudinal-differential phase and (d) the phase velocity excess of the spot of Arago generated by two different disc sizes: the solid line for 4- $\mu \mathrm{m}$ disc and the dashed line for $10-\mu \mathrm{m}$ disc. Markers represent the experimental data for a $4-\mu \mathrm{m}$ disc (open circles) and 10 $\mu \mathrm{m}$ disc (asterisks). The metallic disc is placed in the $z=0 \mu \mathrm{m}$ plane.

On axis, there is an initial phase delay just behind the disc, which is originated from the optical path difference between the diffracted wave and the unperturbed plane wave. This delay equals to the disc radius $r$, which is the distance the light has to travel to the centre of the disc. Far away from the disc at large $z$ values, the path difference between the diffracted wave and the unperturbed plane wave becomes very small and finally disappears. Because at infinity the phase delay is not detectable this phase shift can be interpreted as superluminal wavefront propagation ${ }^{8}$. The phase delay and on-axis phase evolution can be calculated by considering the optical path difference between the diffracted wave and the 
unperturbed plane wave. To do so, we subtract the propagation distance of the plane wave that is just $z$ from the path length of a ray coming from the rim of the disc that travels $\left(z^{2}+r^{2}\right)^{1 / 2}$. The optical path difference becomes:

$$
O P D_{\text {plane wave-diffracted wave }}=\sqrt{z^{2}+r^{2}}-z,
$$

At the disc surface when $z=0 \mu \mathrm{m}$, Eq. (2) confirms the initial phase delay of $r$. In order to examine the superluminal behavior, we derive the phase velocity difference $(\Delta v)$ caused by such phase delays compared with that of the plane wave by differentiating Eq. (2) with respect to time [using $z=c \cdot t]$. We find

$$
\Delta v=c\left(\sqrt{1+\frac{r^{2}}{z^{2}}}-1\right)
$$

with the speed of light $c$ and time t. Normalizing Eq. (3) leads to the phase velocity excess, $(v-c) / c$. The experimental counterpart is obtained by dividing the measured on-axis longitudinal-differential phase by $z$, which leads to the same result as normalizing Eq. (3) and gives

$$
\frac{O P D_{\text {planewave-diffractedwave }}}{z}=\frac{\sqrt{z^{2}+r^{2}}-z}{z}=\sqrt{1+\frac{r^{2}}{z^{2}}}-1=\frac{\Delta v}{c}
$$

Figures 3(c) and 3(d) show the experimental and theoretical axial phase shift and phase velocity excess for the spot of Arago, which is generated by different disc sizes. The solid line stands for a $4-\mu \mathrm{m}$ disc and the dotted line for a $10-\mu \mathrm{m}$ disc. Open circles and asterisks represent the experimental data for $4-\mu \mathrm{m}$ and $10-\mu \mathrm{m}$ discs, respectively. The experimental on-axis longitudinal-differential phase and the corresponding phase velocity excess $[(v-c) / c]$ show excellent agreement with the analytical values obtained from Eqs. (2) and (4). For example, for the 4- $\mu \mathrm{m}$ disc, Fig. 3(c) clearly shows the initial phase delay $r=2 \mu \mathrm{m}(=\sim 3 \lambda)$ and the excess of the speed of light $c$ close to the disc. The case of the plane wave, when no phase shift and therefore no excess of $c$ are present, leads to 0 in both plots shown in Figs. 3(c) and 3(d). Such a phase velocity excess gradually diminishes and falls to zero in the far field, for example (4- $\mu \mathrm{m}$ disc), it exceeds just $2 \%$ of $c$ at position $z=10 \mu \mathrm{m}$. When the size of disc gets larger, the light fields near the obstacle are difficult to measure due to extremely low intensity in the center of the disc. The longitudinal-differential phase near the $10-\mu \mathrm{m}$ disc $[z=0-4 \mu \mathrm{m}]$ is therefore not properly resolved. Nevertheless the measurements are in very good agreement with theoretically predicted values calculated by analytical equations. Such longitudinal-differential phase data allow directly visualizing the on-axis superluminal phase propagation of the spot of Arago, which is localized in the space directly behind the obstacle.

\section{CONCLUSIONS}

We experimentally demonstrated for the first time the direct imaging of axial phase evolution of confined light fields after interaction with microstructures by a new type of interferometric approach, which is termed longitudinaldifferential interferometry. Longitudinal-differential interferometry is implemented in the basement of the HRIM setup, which records the 3D field distributions in amplitude and phase. The measured longitudinal-differential phase distributions directly visualize and quantity the phase difference compared to the plane wave propagating out of the object, which act as the in situ self-reference. Two examples of light interactions with microstructures, which lead to a strong confinement, are studied by the longitudinal-differential-mode HRIM. First, the measurement of a dielectric microsphere shows the Gouy phase anomaly, i.e. $\pi$ shift, in the photonic nanojet. Second, the spot of Arago at low FN has been investigated. The measured longitudinal-differential phase proves the initial phase delay of the disc radius $r$ and the excess of the speed of light $c$. This explains the on-axis local superluminal phase propagation. Overall measurements show excellent agreement with the theoretical values. Those experiments verify the performance of proposed longitudinal-differential interferometry, which is suitable for various kinds of micro- and nano-structures, e.g., fine gratings, small-size microlenses, and amplitude or phase objects.

\section{ACKNOWLEDGMENT}

The research leading to these results has received funding from the European Community's Seventh Framework Programme FP7-ICT-2007-2 under grant agreement No. 224226. 


\section{REFERENCES}

[1] Gouy, L. G., "Sur une propriété nouvelle des ondes lumineuses," Comptes rendus hebdomadaires des séances de l'Académie des Sciences 110, 1251 (1890).

[2] Richards, B. and Wolf, E., "Electromagnetic Diffraction in Optical Systems. II. Structure of the Image Field in an Aplanatic System," Proc. R. Soc. London Ser. A 253, 358-379 (1959).

[3] Foley, J. T. and Wolf, E., "Wave-front spacing in the focal region of high-numerical-aperture systems," Opt. Lett. 30, 1312-1314 (2005).

[4] Visser, T. D. and Wolf, E., "The origin of the Gouy phase anomaly and its generalization to astigmatic wavefields," Opt. Commun. 283, 3371-3375 (2010).

[5] Pang, X., Visser, T. D. and Wolf, E., "Phase anomaly and phase singularities of the field in the focal region of highnumerical aperture systems," Opt. Commun. 284, 5517-5522 (2011).

[6] Subbarao, D., "Topological phase in Gaussian beam optics," Opt. Lett. 20, 2162-2164 (1995).

[7] Kim, M.-S., Scharf, T., Mühlig, S., Rockstuhl, C. and Herzig, H. P., "Gouy Phase Anomaly in Photonic Nanojets," Appl. Phys. Lett. 98, 191114 (2011).

[8] Vasnetsov, M., Pas'ko, V., Khoroshun, A., Slyusar, V. and Soskin, M., "Observation of superluminal wave-front propagation at the shadow area behind an opaque disk," Opt. Lett. 32, 1830-1832 (2007).

[9] Kim, M.-S., Scharf, T., Etrich, C., Rockstuhl, C. and Herzig, H. P., "Longitudinal-differential interferometry: Direct imaging of axial superluminal phase propagation," accepted in Opt. Lett. (December, 2011).

[10]Heifetz, A., Kong, S.-C. , Sahakian, A. V., Taflove, A. and Backman, V., "Photonic Nanojets," J. Comput. Theor. Nanosci. 6, 1979-1992 (2009).

[11] Hecht, E., [Optics, $2^{\text {nd }}$ ed.], Addison Wesley, chap. 10 (1987).

[12] Harvey, J. E. and Forgham, J. L., "The Spot of Arago - New Relevance for an Old Phenomenon,” Am. J. Phys. 52, 243-247 (1984).

[13] Chauvat, D., Emile, O., Brunel, M. and Floch, A. L., "Direct measurement of the central fringe velocity in Youngtype experiments," Phys. Lett. A 295, 78-80 (2002).

[14] Mignai, D., Ranfagni, A. and Ruggeri, R., "Observation of Superliminal Behavoiors in Wave Propagation," Phys. Rev. Lett. 84, 4830-4833 (2000).

[15] Malacara, D., [Optical Shop Testing, $3^{\text {rd }}$ ed.], WILEY, chap. 16 (2007).

[16] Kim, M.-S., Scharf, T. and Herzig, H. P., "Amplitude and Phase Measurements of Highly Focused Light in Optical Data Storage Systems,” Jpn. J. Appl. Phys. 49, 08KA03 (2010).

[17] Kim, M.-S., Scharf, T. and Herzig, H. P., "Small size microlenes characterization by Multiwavelength High Resolution Interference Microscopy," Opt. Express 18, 14319-14329 (2010).

[18] Kim, M.-S., Scharf, T., Mühlig, S., Rockstuhl, C. and Herzig, H. P., "Engineering photonic nanojets,” Opt. Express 19, 10206-10220 (2011).

[19] Kim, M.-S., Scharf, T., Haq, M. T., Nakagawa, W. and Herzig, H. P., "Subwavelength-size solid immersion lens," Opt. Lett. 36, 3930-3932 (2011).

[20] Kim, M.-S., Scharf, T., Mühlig, S., Rockstuhl, C. and Herzig, H. P., "Gouy Phase Anomaly in Photonic Nanojets," Appl. Phys. Lett. 98, 191114 (2011).

[21] Rockstuhl, C., Märki, I., Scharf, T., Salt, M., Herzig, H. P. and Dändliker, R., "High Resolution Interference Microscopy: A Tool for Probing Optical Waves in the Far-Field on a Nanometric Length Scale," Current Nanoscience 2, 337-350 (2006).

[22] Schwider, J., Burow, R., Elssner, K.-E., Grzanna, J., Spolaczyk, R. and Merkel, K., "Digital wave-front measuring interferometry: some systematic error sources," Appl. Opt. 22, 3421-3432 (1983).

[23] Hariharan, P., Oreb, B. F. and Eiju, T., "Digital phase-shifting interferometry: a simple error-compensating phase calculation algorithm," Appl. Opt. 26, 2504-2506 (1987).

[24] G. Mie, "Beiträge zur Optik trüber Medien, speziell kolloidaler Metallösungen,” Ann. d. Physik, 25, 377 (1907).

[25] Van de Hulst, H. C., [Light Scattering by Samll Particles], Dover, chap. 9 (1981). 